\title{
Viability of pHEMA Hydrogels as Coating in Human Synovial Joint Prosthesis
}

\author{
V.P. Bavaresco $^{\mathrm{a} 1}$, C.A.C. Zavaglia $^{\mathrm{a} 2}$, S.M. Malmonge ${ }^{\mathrm{b} 3}$, M.C. Reis $^{\mathrm{c} 4}$ \\ ${ }^{a}$ Department of Materials Engineering, College of Mechanical Engineering \\ Universidade Estadual de Campinas (UNICAMP) \\ Cx. P. 6122, 13081-970 Campinas - SP, Brazil \\ ${ }^{\mathrm{b}}$ FECQ/Universidade Metodista de Piracicaba, Piracicaba - SP, Brazil \\ ${ }^{\mathrm{c} C T C / U n i v e r s i d a d e}$ Estadual de Campinas (UNICAMP), Campinas - SP, Brazil
}

Received: February 14, 2002; Revised: August 22, 2002

\begin{abstract}
In artificial joints, the bone part is usually substituted by a metallic component with high corrosion and strength resistance while the articular cartilage is replaced by a polymer. Use of thin layer of a compliant material acting as a bearing surface in human replacement joints has recently generated considerable interest. This work analyses the coating of a solid porous substrate of Ultra High Molecular Weight Polyethylene (UHMWPE) with a poly (2-hydroxyethyl methacrylate) (pHEMA) and two sIPN-type blends: pHEMA-cellulose acetate butyrate (CAB) and pHEMA-poly (ethyl methacrylate) (PEM) using 5.0\% (w/w) of the crosslinking agent and $11.0 \%$ $(\mathrm{w} / \mathrm{w})$ of the linear reinforcing polymer. The wear resistance of the coating materials was evaluated in a TRI PIN ON DISK type equipment and the damage extension was characterized by Scanning Electron Microscopy (SEM). Preliminary qualitative tests were performed with the goal to identifying the hydrogels show the minimal required properties concerning wear strength. The pHEMA coating was completely destroyed during the first wear cycles, characterizing its low shear strength. By the other hand, after the complete experiment, both pHEMA-CAB and pHEMAPEM blends showed a slightly improvement of abrasive and adhesive wear. This result indicates that the studied blends are promising materials to be used as compliant surfaces in joint prosthesis.
\end{abstract}

Keywords: UHMWPE, hydrogel pHEMA, joint prosthesis

\section{Introduction}

The study of lubrication, friction and wear of natural and artificial joints has allowed an increase in the knowhow on the action of natural joints and the cause of failure. The association of biochemical and biomechanical factors contribute to the interfacial collapse implant/tissue depending on the type of substituted tissue. When the surface wear increases, the artificial joint moves altering the distribution of local stress, thus accelerating interfacial collapse. Besides, several adverse reactions may occur between the material and the physiologic medium, especially when there is a delivery of particle debris in the joint. This knowledge supplies information for the development of new materials to be used as surface in artificial articular cartilage.

Ultra High Molecular Weight Polyethylene (UHMWPE) has been the most used material as bearing surfaces in ar- throplasty joints, because of its wear resistance and excellent biocompatibility. However, it has recently become evident that the wear of UHMWPE is one of the important factors in the failure of joint prostheses ${ }^{1-5}$.

Use of thin layer of compliant material acting as a bearing surface in human replacement joints has recently generated considerable interest. The theory predicts that, contrary to the already existing joint prosthetic projects, the joint that has a layer of a compliant material on the surface can produce a lubrication film between the surfaces. The produced lubrification film has a low coefficient of friction, obtaining a reduction of friction wear. Some researchers have shown that in the case of an artificial joint with very hard surfaces, there is no production of a fluid film with elastohydrodynamic behavior, similar to natural synovial joint ${ }^{6-9}$.

*e-mail: ${ }^{1}$ petrilli@fem.unicamp.br, ${ }^{2}$ zavagl@ fem.unicamp.br, ${ }^{3}$ smmalmon@unimep.br, ${ }^{4}$ mcreis@unicamp.br 
Polymeric hydrogel is a very interesting material to be used as compliant material in artificial joints. Hydrogels based on hydroxyalkyl methacrylates such as poly (2-hydroxyethyl methacrylate) (pHEMA) have been used for soft tissue prostheses due to their biocompatible characteristics, high permeability to small molecules (i.e. tissue metabolites), high hydrophilicity and their soft consistency. However, in this case the artificial articular cartilage, the hydrogels should present a high amount of water for the transport of nutrients, the mechanical properties are inadequate and are not able to resist at the high stress levels the joint is submitted ${ }^{10-12}$. Hydrogels with good mechanical performance have been obtained by the association of different materials, such as the incorporation of a hydrophobic component in a polymeric network ${ }^{5,6,8,13,14}$. A great variety of linear reinforcing polymers such as polymetacrylates (i.e. polyethyl methacrylate - PEM), polyuretanes and modified cellulose (i.e. cellulose acetate butyrate - $\mathrm{CAB}$ ) have been studied for use in the semi-interpenetrating polymer networks, in order to produce hydrogels with improved mechanical properties ${ }^{15}$.

Many workers have shown that compliant layers improve the behavior of artificial joints but, the success of this system depends on the integrity and durability of surface during the prosthesis life $\mathrm{e}^{6,9}$.

The objective of the laboratory wear testing is the prediction of the clinical performance of bearing materials and designs. The intrinsic characteristics of the polymeric materials as well as wear testing conditions are important to define and to study the material wear resistance.

In this work, the viability of using pHEMA hydrogels and sIPN from pHEMA hydrogel reinforced with CAB or PEM as compliant surface in human synovial joint prosthesis was investigated. Solid porous substrate of UHMWPE was coated with polymer hydrogels and was submitted to the wear testing in a TRI-PIN-ON-DISK equipment. The purpose of the preliminary wear testing was to verify the wear resistance of these hydrogels, by qualitative way.

\section{Materials}

Solid porous substrates of UHMWPE obtained in cylinder form with $2.5 \times 10^{-2} \mathrm{~m}$ in diameter and $1.5 \times 10^{-2} \mathrm{~m}$ in height, were coated with pHEMA hydrogel and sIPN blends pHEMA-CAB and pHEMA-PEM.

The hydrogels were obtained from a mixture of 2-hydroxyetlyl metacrylate (HEMA) (Aldrich), benzoil peroxide $(0.5 \% \mathrm{w} / \mathrm{w})$ as an initiator of polymerization, and trimethylol propane trimethacrylate (TMPTMMA) (Retilox) $(5 \% \mathrm{w} / \mathrm{w})$ as a crosslinking agent. The sIPN blends were obtained using pHEMA hydrogel with $11 \%$ of linear reinforcing polymer - cellulose acetate butyrate (CAB) (Aldrich) (Mn 70.000) or poly (ethyl methacrylate) (PEM) (Aldrich)
(Mw 515.000) and 5\% (w/w) of TMPTMMA as crosslinking agent. The porous substrates were immersed in a mixture of monomer, initiator and crosslinking agent. The system went through thermal polymerization for $4 \mathrm{~h}\left(70-85^{\circ} \mathrm{C}\right)$. After that, it was placed in water for the removal of the residual monomer and initiator. Thus, the hydrogels were formed on the surface and inside the pores of the substrates, assuring its mechanical fixation.

\section{Wear test using a TRI-PIN-ON-DISK equipment}

The wear testing was conducted on TRI-PIN-ON-DISK equipment ${ }^{16}$ using a stainless steel $316 \mathrm{~L}$ (ASTM) disk as a counterface, in the presence of distilled water with a sliding speed of $0.34 \mathrm{~m} / \mathrm{s}$, contact tension of $1 \mathrm{MPa}$ and total distance of $2500 \mathrm{~m}$ or 8.842 cycles.

The counterface disks were prepared by polishing them in polishing equipment using abrasive disks with 100, 200, 320, 400, 600 and 1200 mesh, in this sequence, under water circulation. The disks were washed, first with detergent and water and then with absolute ethyl alcohol. After that, the solvent was evaporated in hot air flow.

\section{Scanning Electron Microscopy - SEM}

The morphology of the samples submitted to the wear test was determined by SEM (MEV JEOL JXA 860A). The samples were gold sputtered, using Sputter Coater BALTEC SCD equipment. Results and discussions.

Figure 1 shows the result of the wear testing from the solid porous substrate of UHMWPE coated with pHEMA hydrogel with 5\% of TMPTMMA. The pHEMA coating was completely destroyed in the first wear cycles. The analy-

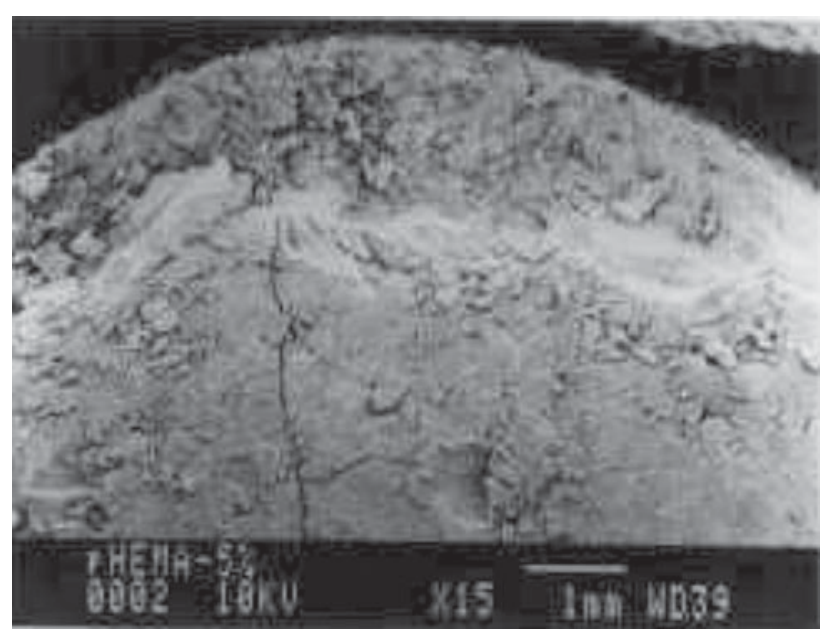

Figure 1. SEM micrograph of UHMWPE substrate with pHEMA hydrogel. 
sis of the surface allowed verifying that hydrogel was absorbed in the porous substrate.

Figures 2 to 4 show the micrographic images of the porous substrate covered with the pHEMA-CAB blend and pHEMA-PEM blend. It was observed that the coating resisted to the complete wear cycles without being destroyed.

Figure 2 shows the damaged area of the pHEMA-CAB blend surface. The small depth can be an indication of the adhesive process.

Figure 3a shows the onset of the wear on the surface of the pHEMA-CAB blend. Figure $4 \mathrm{~b}$ shows the continuity of the wear forming cavities on the surface of the material, characterizing wear by abrasion. The arrow indicates the direction of friction sliding.

Figure 4 shows the same area of the start-up of adhesive wear presented in Fig. 3a with amplified form. The photo shows the area where the hydrogel was pulled up by adhesion, allowing the formation of cavities that are characteristic of abrasive wear.

The pHEMA-PEM blend presented a more damaged surface with a larger number of wear areas. Figures 5 and 6 shows the occurrence of adhesive and abrasive wear.

Figure 5a shows free fragments that may have been generated by the abrasive wear. The observed cavities clearly characterize the occurrence of abrasive wear. Figure $5 b$ shows a detail of the same area.

Figure 6 shows the damaged surface of the pHEMAPEM blend. A fissure, generated due to the shear strength caused by friction forces, can be observed.

Therefore, pHEMA-CAB blend showed the best results under the conditions in which the wear testing was carried out. Although CAB used in this study has a smaller molecular weight than PEM, its molecular structure presents voluminous acetate and butyrate groups that act as physical

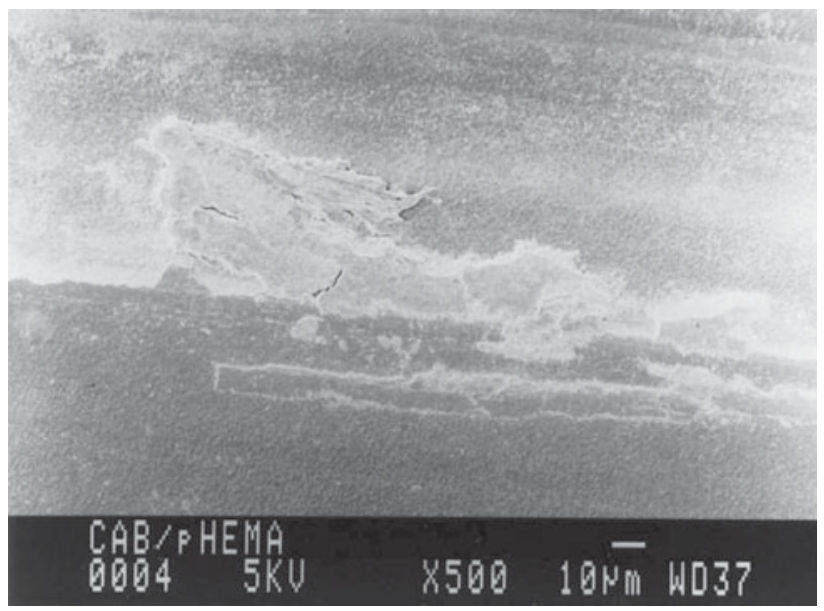

Figure 2. SEM worn surface of the pHEMA-CAB blend.
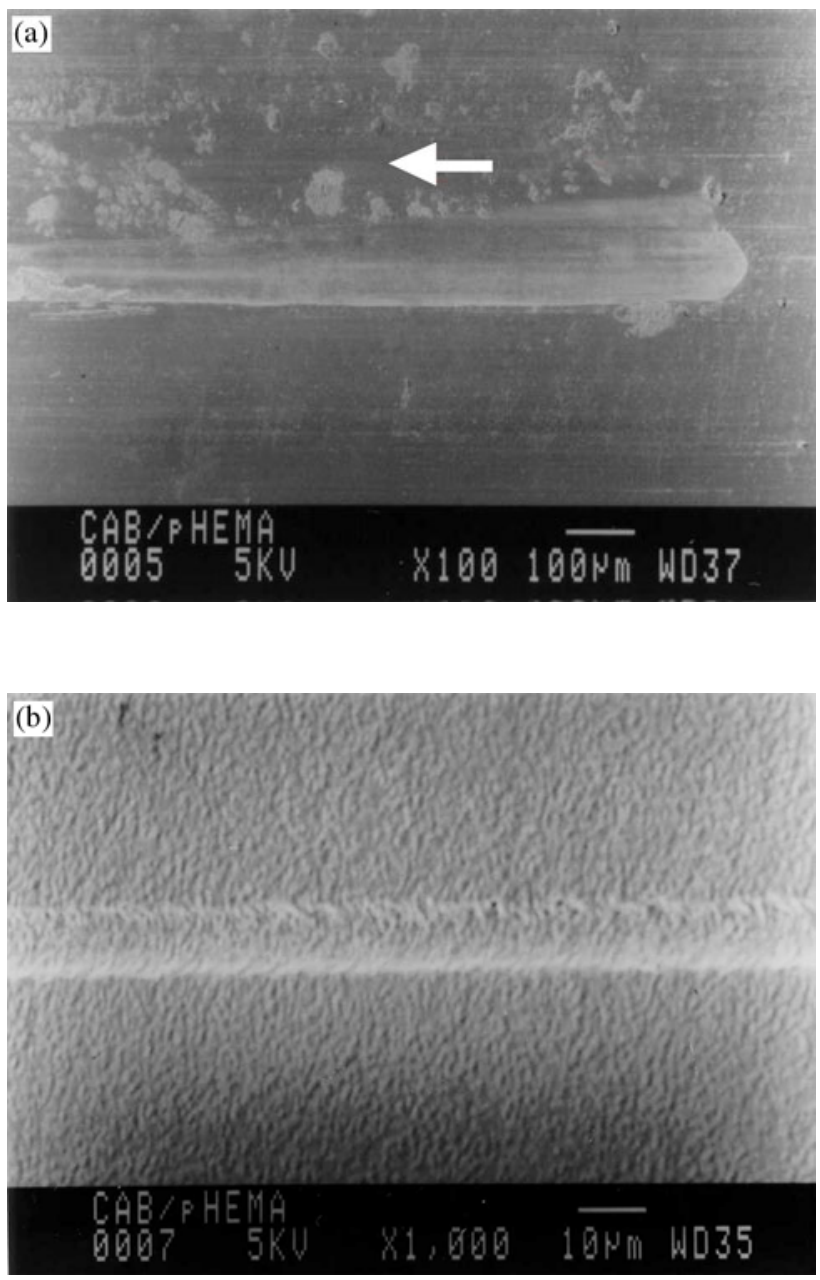

Figure 3. SEM from the worn surface of the pHEMA-CAB blend, (a) starting wear by abrasion, (b) abrasive wear.

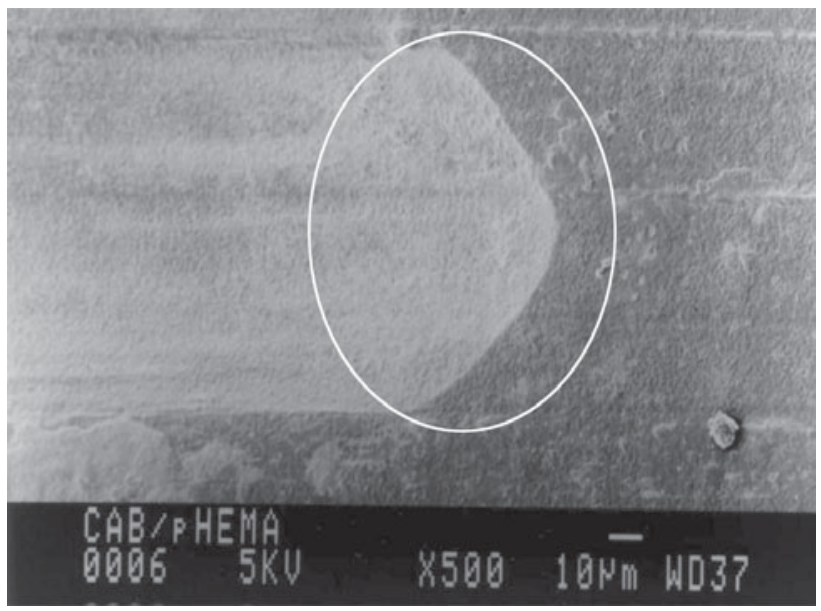

Figure 4. SEM worn surface of the pHEMA-CAB blend. 

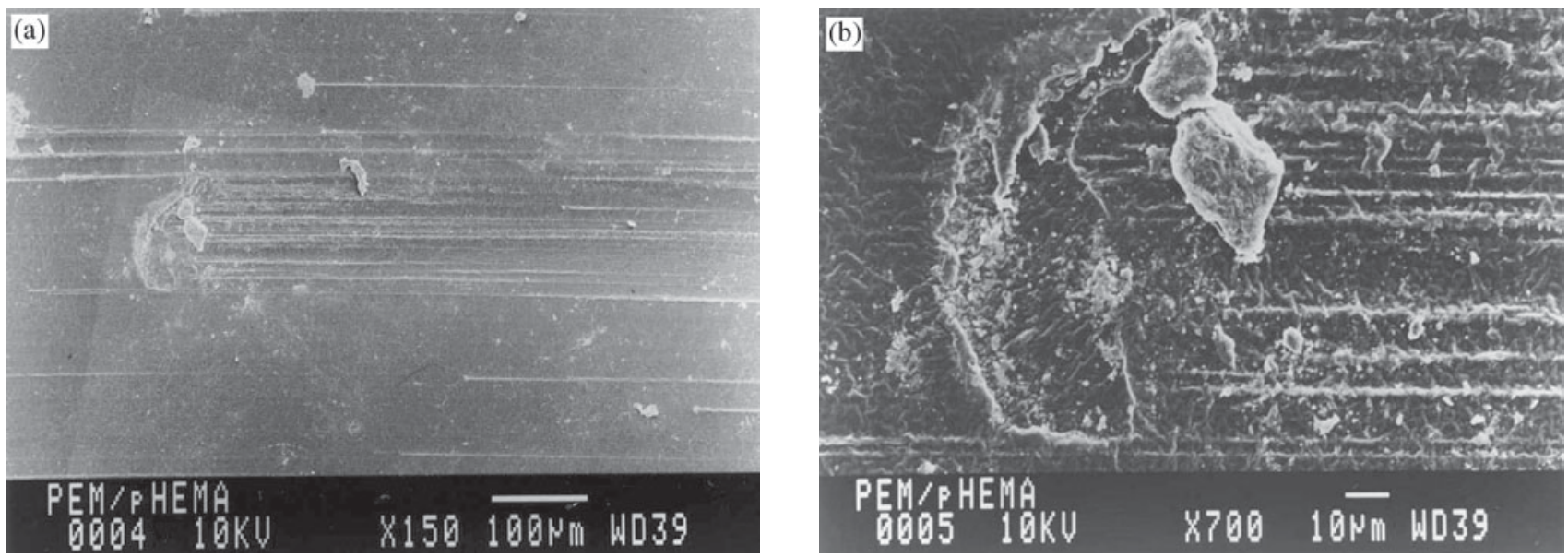

Figure 5. SEM worn surface of the pHEMA-PEM blend (a) and (b) detail of.

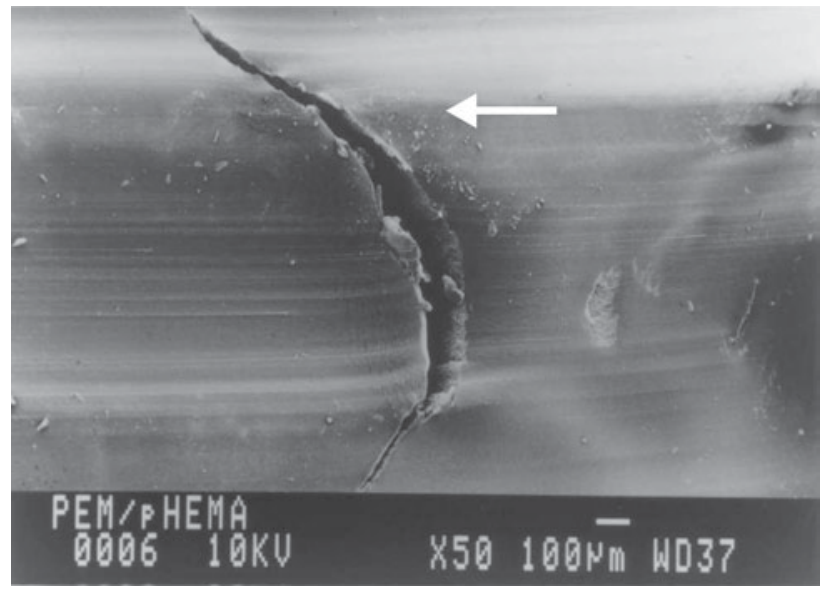

Figure 6. SEM of the pHEMA-PEM blend worn surface. The arrow indicates the direction of the movement of friction sliding.

barriers hindering the movement of PEM among the networks.

Soft or compliant materials are easily destroyed by adhesion, promoting the verified damage on the pHEMA hydrogel. As hardness of the hydrogel increased through the synthesis of the sIPN blends, a significant wear resistance improvement was observed.

\section{Conclusion}

The pHEMA hydrogel with 5\% (w/w) of TMPTMMA as a crosslinking agent did not resist to the wear testing. The pHEMA-CAB blend and pHEMA-PEM blend, synthesized with 5\% (w/w) of TMPTMMA and $11 \%(\mathrm{w} / \mathrm{w})$ of linear reinforcement polymer, showed only slight abrasive and adhesive wear. The results obtained in this study suggest the improving of the mechanical properties of pHEMA hydrogel by reinforcing the network with $\mathrm{CAB}$ and PEM polymers.

\section{Acknowledgments}

To FAPESP and Finep-Pronex for the financial support.

\section{References}

1. Wang, A.; Essner, A.; Polineni, V.K.; Stark, C.; Dumbleton, J.H. Tribology International, v. 31, p. 17-33, 1998.

2. Learmonth, I.D.; Cunningham, J.L. Proc Inst. Mech. Engrs., part H, v. 211, p. 49-57, 1997.

3. Lewis, G. Polyethylene wear in total hip and knee arthroplasties, Department of Mechanical Engineering, The University of Memphis, Memphis, Tennessee, p. 55-75, 1997.

4. Ambrosio, L.; Carotenuto, G.; Marletta, G.; Nicolais, L.; Scandurra, A. Journal of Materials Science: Materials in Medicine, v. 7, p. 723-729, 1996.

5. McGee, M.A.; Howie, D.W.; Neale, S.D.; Haynes, D.R.; Pearcy, M.J. Proc. Inst. Mech. Engrs, part. H, v. 211, p. 65-72, 1996.

6. Bigsby, R.J.A.; Auger, D.D.; Jin, Z.M.; Dowson, D.; Hardaker, C.S.; Fisher, J. Journal of Biomechanics, v. 31, p. 363-369, 1998.

7. Hall, R.M.; Unsworth, A. Biomaterials, v.18, p.1017-1026, 1997.

8. Caravia, L.; Dawson, D.; Fisher, J. Journal of Materials Science: Materials in Medicine, v. 4, p. 515-520, 1993.

9. Blamey, J.; Rajan, S.; Unsworth, A.; Dawber, R. Journal Biomedical Engineering, v. 13, p. 180-184, 1991.

10. Netti, P.A.; Shelton, J.C.; Revell, P.A.; Pirie, C.; Smith, S.; Ambrosio, L.; Nicolais, L.; Bonfield, W. Biomaterials, v. 14, p. 1098-1104, 1993.

11. Kudela, V. in: Jacqueline, I.K., (ed) Encyclopedia of Polymer Science and Engineering, p. 783-807, 1990.

12. Ratner, B.D.; Hoffman, A.S. ACS Symposium Series, v. 31, p. $1-36,1976$

13. Malmonge, S.M.; Zavaglia, C.A.C. Polímeros - Ciência e Tecnologia, v. 2, p. 22-29, 1997.

14. Corkhill, P.H.; Tighe, B.J. Polymer, v. 21, p. 1526-1537, 1990.

15. Corkhill, P.H.; Trevett, A.S.; Tighe, B.J. Proc. Inst. Mech. Engrs. Part H: Journal of Engineering in Medicine, v. 204, p. 147$155,1989$.

16. Reis, M.C.; Zavaglia, C.A.C. Brazilian Journal of Biomedical Engineering, v. 15, p. 31, 1999. 